14

\title{
Связь явлений переноса с характеристиками кластерной структуры воды
}

\author{
() А.В. Полянская, ${ }^{2}$ А.М. Полянский, ${ }^{1}$ В.А. Полянский ${ }^{3}$ \\ ${ }^{1} \mathrm{OOO} \mathrm{„НПК} \mathrm{электронные} \mathrm{и} \mathrm{пучковые} \mathrm{технологии“,}$ \\ 198188 Санкт-Петербург, Россия \\ ${ }^{2}$ Санкт-Петербургский политехнический университет Петра Великого, \\ 195251 Санкт-Петербург, Россия \\ ${ }^{3}$ Институт проблем машиноведения РАН, \\ 199178 Санкт Петербург, Россия \\ e-mail: ampol@electronbeamtech.com
}

Поступило в Редакцию 15 января 2018 г.

В окончательной редакции 25 декабря 2018 г.

Принято к публикации 29 декабря 2018 г.

С помощью статистического подхода сделаны оценки размера кластеров воды и числа свободных молекул, участвующих в явлениях переноса. На базе экспериментальных данных по температурной зависимости вязкости и плотности воды определены размеры кластеров и число молекул в них, количество свободных молекул воды и длины их свободного пробега. Сделан вывод о том, что температура $36.6^{\circ} \mathrm{C}$ является особой точкой в диапазоне $0-100^{\circ} \mathrm{C}$. При температурах выше этого значения происходит скачкообразное уменьшение энергии связи молекулы с кластером и резкое возрастание концентрации свободных молекул. Количественные данные сравнены с параметрами сверхпластичного состояния титана. Рассмотрен обмен веществ на примере функционирования эритроцитов крови в организме взрослого человека.

DOI: $10.21883 /$ JTF.2019.06.47647.367-18

\section{Введение}

Новые научные приоритеты в материаловедении, приборостроении, энергетике и науке о человеке связаны с размерностью структур от десятых долей до десятков нанометров. Около 100 лет тому назад при исследовании каталитической активности платины, имеющей поликристаллическую структуру, был установлен оптимальный размер ее кристаллов, равный 40-50 А, при котором каталитическая активность становится максимальной [1]. Исследования были выполнены с использованием дифракции рентгеновских лучей.

В. Стюарт [2] применил метод дифракции рентгеновских лучей к исследованию структуры ряда жидкостей. Было обнаружено сходство между рентгенограммами мелкокристаллических тел и ряда жидкостей. Я.И. Френкель в своей монографии [3] обобщил экспериментальные данные, предложив новую интерпретацию характера теплового движения частиц в жидкостях. Согласно [3], основная часть атомов находится в „оседлых состояниях“, в которых атом совершает колебательное движение. Время жизни частицы в таком состоянии существенно больше, чем период колебаний атомов в твердом теле, составляющий порядка $10^{-13} \mathrm{~s}$. Затем атом покидает свое „оседлое состояние“ и оказывается в ближайшем „оседлом состоянии“.

Таким образом, явления переноса в жидкостях определяются тепловым движением свободных атомов между „оседлыми состояниями“. Диффузия, теплопроводность и вязкое трение определяются средней длиной свобод- ного пробега атомов жидкости. Этот подход последовательно применялся разными авторами для описания явлений диффузии и теплопроводности в жидкостях.

Вода обладает уникальными свойствами. В 1891 г. В. Рентген впервые предложил рассматривать ее как двухкомпонентную жидкость [4]. В последних работах, опубликованных на эту тему [5-15], для изучения свойств воды использованы самые современные методы физических исследований. Тонкие эксперименты по определению электрических и магнитных свойств кластеров, объединяющих молекулы воды, позволили определить количественный состав кластеров: от 3 до 50 молекул воды в каждом. В этом же диапазоне по числу молекул находятся результаты теоретических исследований с использованием методов молекулярной динамики [14]. Малоугловое рассеяние излучения лазера привело к обнаружению „макрокластеров“, радиус которых составляет от 0.5 до $6 \mu \mathrm{m}[12,13]$. Природа таких неоднородностей до конца не выяснена. По мнению одних авторов - это кластеры, образованные молекулами воды, по мнению других - стабильные микропузырьки растворенных газов (бабстоны).

Влияние кластерной наноструктуры воды на явления переноса ионов и их химическую активность исследовалось в [16-19]. Авторами получены оценки частот колебательных степеней свободы кластеров воды, содержащих ионы солей с различным количеством молекул воды. Эти частоты сравниваются с экспериментальными линиями ИК-спектров. На основании сопоставлений сделан вывод о зависимости кластерной структуры от 
химической формулы растворенной соли. Речь идет о кластерах, в составе которых содержится не более 10 молекул воды.

С использованием различных физических методов, включая электронную микроскопию водных пленок при низких температурах, в [20] исследована кластерная структура воды на смачиваемой поверхности твердого тела.

Моделирование кластерной структуры воды методами молекулярной динамики выполнено в работе [21]. Основой причиной образования кластеров считается водородная связь между молекулами. С использованием уравнений молекулярной динамики рассчитывается устойчивость кластеров с количеством молекул от двух до шести.

Не смотря на большой интерес к структуре воды и водных растворов, до сих пор особенности строения воды не имеют единого описания в рамках кинетической теории.

\section{Оценка длины свободного пробега молекул воды}

Согласно классической теории явлений переноса $[22,23]$, длина свободного пробега обратно пропорциональна сечению столкновения атома с „оседлыми состояниями“ и концентрации этих состояний.

Конкретизируем задачу. Пусть „оседлое состояние“ представляет собой некую ассоциацию атомов или молекул жидкости, назовем ее кластером радиуса $r_{0}$. При размере кластера в несколько десятков $\AA$, как было установлено в [2], он будет содержать тысячи атомов. Следовательно, подвижность кластера будет чрезвычайно мала по сравнению с подвижностью отдельных атомов или молекул, и вкладом кластеров в явлениях переноса можно пренебречь. Такой подход является универсальным для любых жидкостей, в том числе и для воды [3,24].

Перенос импульса в жидкости определяется коэффициентом вязкости $\mu$ :

$$
\mu=\frac{1}{3} v \cdot \rho \cdot \lambda,
$$

где $v-$ средняя скорость хаотического теплового движения молекул воды при данной температуре $T, \rho-$ плотность воды, $\lambda-$ средняя длина свободного пробега молекул воды.

Длина свободного пробега обратно пропорциональна величине $\sigma-$ сечения столкновения молекулы с кластером радиуса $r_{0}-$ и концентрации кластеров $n_{\mathrm{cl}}$.

Схема взаимодействия молекулы воды с кластером радиуса $r_{0}$ приведена на рис. 1 .

Можно предположить, что при температуре $T=0 \mathrm{~K}$ все молекулы воды находятся внутри кластеров.

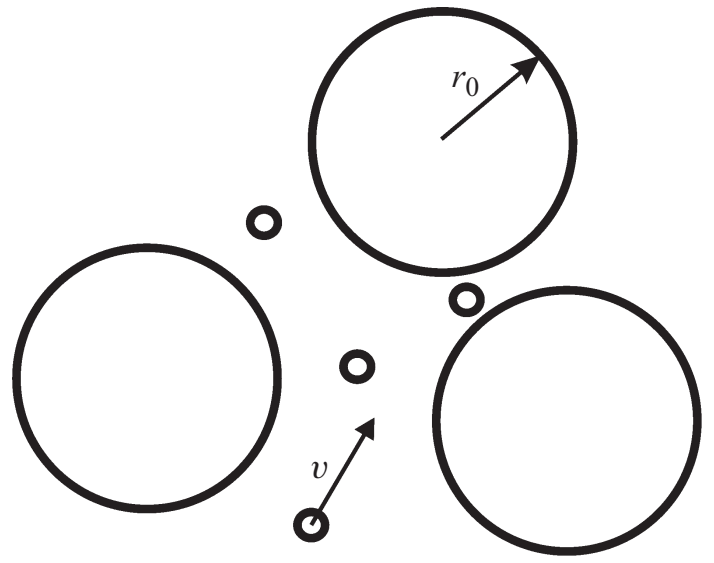

Рис. 1. Модель столкновения молекулы воды с кластером радиуса $r_{0}, v-$ средняя скорость теплового движения молекулы воды.

По определению средняя концентрация молекул $n$ связана с плотностью материала $\rho$ соотношением

$$
n=\frac{\rho}{m_{0}}=\frac{\rho}{M} \cdot N_{A},
$$

где $m_{0}$ - масса молекулы, $M$ - масса 1 грамм-моля вещества, $N_{A}=6.02 \cdot 10^{23}$ - число Авогадро. Подставляя значения $\rho, M$ и $N_{A}$, получим $n=3.344 \cdot 10^{22} \mathrm{~cm}^{-3}$.

Средние расстояния $d$ между молекулами воды в $[\mathrm{cm}]$ определяются формулой

$$
d=\sqrt[3]{\frac{1}{n}}=\sqrt[3]{\frac{M}{\rho \cdot N_{A}}} .
$$

Среднее расстояние между молекулами при температурах, близких к $4{ }^{\circ} \mathrm{C}$, после подстановки численных значений в формулу (3), будет равно $d=0.31028 \cdot 10^{-7} \mathrm{~cm}$.

Число молекул воды $N_{0}$ в кластере радиуса $r_{0}$ определяется отношением объема кластера к объему $d^{3}$, приходящемуся на 1 молекулу в кластере:

$$
N_{0}=\frac{4}{3} \pi \cdot \frac{r_{0}^{3}}{d^{3}} .
$$

Концентрация кластеров $n_{\mathrm{cl}}$, при условии, что их размеры примерно одинаковы и все молекулы заселяют их равномерно, будет равна

$$
n_{\mathrm{cl}}=\frac{n}{N_{0}}=\frac{3 \cdot n \cdot d^{3}}{4 \pi \cdot r_{0}^{3}} .
$$

Если объем, приходящийся на 1 молекулу, равен $d^{3}$, а концентрация молекул воды равна $n$, то справедливо соотношение $n \cdot d^{3}=1$. С учетом этого соотношения $n_{\mathrm{cl}}$ будет равно

$$
n_{\mathrm{cl}}=\frac{3}{4 \pi \cdot r_{0}^{3}} \text {. }
$$

При температуре, отличной от абсолютного нуля, подавляющая часть молекул воды будет сосредоточена 
Таблица 1. Радиус кластера и длина свободного пробега молекул воды с учетом ее кластерной структуры при трех различных температурах

\begin{tabular}{c|c|c}
\hline$T,{ }^{\circ} \mathrm{C}$ & $r_{0}, \AA$ & $\lambda, \AA$ \\
\hline 0 & 90.6 & 78.0 \\
36.6 & 31.8 & 21.6 \\
100 & 12.2 & 6.60
\end{tabular}

в кластерах, поэтому с некоторой погрешностью можно считать, что выполняются все зависимости, полученные выше.

Согласно [22,23], средняя длина свободного пробега молекул воды равна

$$
\lambda=\frac{1}{\sqrt{2} \sigma \cdot n_{\mathrm{cl}}}
$$

где $\sigma$ - эффективное сечение столкновения, определяемое радиусом кластера $r_{0}$ и радиусом молекулы воды $r$ :

$$
\sigma=\pi\left(r_{0}+r\right)^{2}
$$

Учитывая малость размера молекулы $r$ по сравнению с размером кластерной структуры $r_{0}$, получим

$$
\sigma \cong \pi r_{0}^{2} \cdot\left(1+2 \frac{r}{r_{0}}+\frac{r^{2}}{r_{0}^{2}}\right)
$$

Последним слагаемым в формуле можно пренебречь, как квадратом малой величины. Средняя длина свободного пробега молекулы воды будет равна

$$
\lambda=\frac{1}{\sqrt{2} \pi r_{0}^{2} \cdot\left(1+2 \frac{r}{r_{0}}\right) \cdot n_{\mathrm{cl}}} .
$$

Подставим в полученное выражение значение концентрации кластерных структур (6):

$$
\lambda=\frac{4}{3 \sqrt{2}} \cdot \frac{r_{0}}{\left(1+2 \frac{r}{r_{0}}\right)}=0.94281 \cdot \frac{r_{0}}{1+2 \frac{r}{r_{0}}} .
$$

Полученное значение длины свободного пробега (10) подставим в формулу (1), определяющую значение коэффициента вязкости:

$$
\mu=0.31427 \cdot v \cdot \rho \cdot \frac{r_{0}}{1+2 \frac{r}{r_{0}}} .
$$

С учетом малости отношения $\frac{r}{r_{0}} \ll 1[1,2]$ можно получить оценку для радиуса кластера $r_{0}$ :

$$
r_{0}=3.182 \cdot \frac{\mu}{v \cdot \rho},
$$

где параметры жидкости - средняя скорость теплового движения молекул $v=v(T)$, кинематическая вязкость $\mu=\mu(T)$, и плотность $\rho=\rho(T)$ зависят от температуры.
Таблица 2. Результаты оценки среднего числа молекул в кластере $N_{0}$, числа свободных молекул на каждый кластер $N_{\mathrm{H}_{2} \mathrm{O}}$ и времени „оседлой жизни“ $\tau$ для нескольких температурных точек

\begin{tabular}{c|c|c|c|c|c}
\hline$T,{ }^{\circ} \mathrm{C}$ & $r_{0}, \AA$ & $E, \mathrm{eV}$ & $N_{0}$ & $N_{\mathrm{H}_{2} \mathrm{O}}$ & $\tau, \mathrm{s}$ \\
\hline 0 & 90.6 & 0.22 & $10^{5}$ & 10.4 & $3.9 \cdot 10^{-10}$ \\
$<36.6$ & 31.8 & 0.22 & $4.5 \cdot 10^{3}$ & 1.2 & $3.9 \cdot 10^{-10}$ \\
$>36.6$ & 31.8 & 0.135 & $4.5 \cdot 10^{3}$ & 28.7 & $1.6 \cdot 10^{-11}$ \\
100 & 12.2 & 0.135 & $2.5 \cdot 10^{2}$ & 4.60 & $1.6 \cdot 10^{-11}$
\end{tabular}

В табл. 1 приведены значения величины средней длины свободного пробега молекул воды $\lambda$ и радиуса кластеров $r_{0}$ для трех температурных точек: $0,36.6$ и $100^{\circ} \mathrm{C}$. Вычисления выполнены по формуле (10) с использованием значений $r_{0}$, полученных по формуле (11). Значения $\mu(T)$ и $\rho(T)$ взяты из экспериментальных данных [25]. В диапазоне температур от 0 до $100^{\circ} \mathrm{C} \mu(T)$ принимают значения от 1.8 до $0.28 \mathrm{mPa} \cdot \mathrm{s}$.

Среднее значение расстояния между молекулами воды (жидкое состояние) при нормальных условиях $d=3.1038 \AA$. Таким образом, средняя длина свободного пробега молекул $\mathrm{H}_{2} \mathrm{O}$ становится соизмеримой со средним расстоянием между молекулами только при температуре кипения. В диапазоне температур $0-100^{\circ} \mathrm{C}$ отношение $\lambda / d$ за счет кластерной структуры воды варьирует от 25.0 до $\sim 2.0$ соответственно.

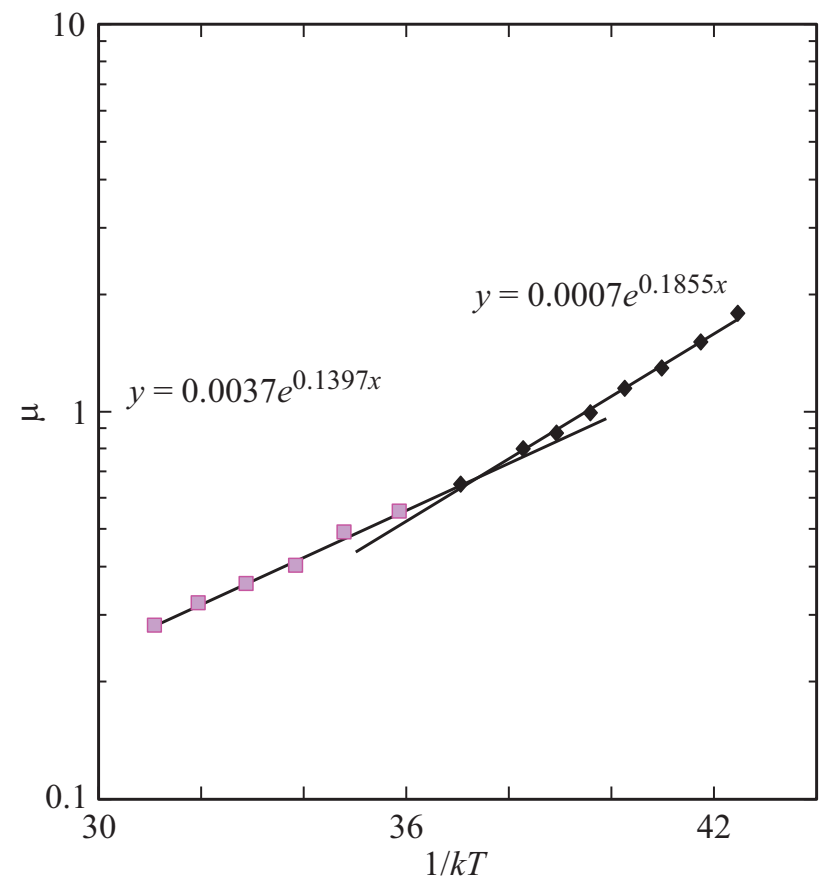

Рис. 2. Зависимость коэффициента вязкости $\mu$ от $1 / k T$ в логарифмическом масштабе. Точками изображены данные [25], сплошной линией - результат линейной аппроксимации. 


\section{Оценка числа свободных молекул воды}

Зависимость коэффициента вязкости от температуры носит активационный характер [3] и может быть представлена в виде

$$
\mu=\mu_{0} \exp \left(-\frac{E}{k T}\right)
$$

где $E$ - энергия связи молекулы с кластером в „оседлом“ состоянии („энергия активации“ молекулы в „оседлом“ состоянии по Я.И. Френкелю [3]).

График зависимости логарифма коэффициента вязкости $\mu$ от $1 / k T$ представлен на рис. 2.

Зависимость для воды можно аппроксимировать двумя прямыми, угол наклона которых определяет энергию связи молекулы воды с кластером. Таким образом, для воды при нормальных условиях, энергия связи имеет два значения:

${ }^{*} E_{1}=0.22 \mathrm{eV}$ в диапазоне температур от 0 до $36.6^{\circ} \mathrm{C}$, ${ }^{*} E_{2}=0.13 \mathrm{eV}$ при температурах от 36.6 до $100^{\circ} \mathrm{C}$.

Точка пересечения двух прямых на графике, представленном на рис. 2, соответствует температуре воды $36.6^{\circ} \mathrm{C}$. Температура $36.6^{\circ} \mathrm{C}$ является фундаментальной характеристикой воды. При этой температуре энергия связи молекулы воды с кластером скачком уменьшается почти в 2 раза. В этой связи почти на порядок сокращается время жизни молекулы воды в кластере, почти в 20 раз возрастает доля молекул, покидающих кластер в каждую секунду. Таким образом, суммарная подвижность молекул воды при температуре $t \geq 36.6^{\circ} \mathrm{C}$ возрастает в сотни раз. Возможно, в этом кроется фундаментальное объяснение функционирования организма человека в рамках представлений о сочетании кластерной и молекулярной структуры воды во всем диапазоне температур от 0 до $100^{\circ} \mathrm{C}$.

В табл. 2 приведены оценки среднего числа молекул в кластере $N_{0}$, числа свободных молекул на каждый кластер $N_{\mathrm{H}_{2} \mathrm{O}}$ и времени „оседлой жизни“ $\tau$ для нескольких температурных точек.

\section{Обсуждение результатов}

В пользу кластерной модели структуры воды говорит недавнее открытие димеров (образование из 2 молекул воды) в атмосфере [9].

Соотношение (11) получено благодаря использованию гипотезы о том, что кластеры (или кристаллиты) при течении жидкости являются твердыми образованиями, а скольжение их друг относительно друга обеспечивается прослойкой свободных молекул воды, характеристики которых ( $v$ и $\lambda)$ содержатся в классической формуле (1). Часть молекул воды находится внутри кластеров, часть - в виде отдельных молекул. Связь молекул с кластером велика $(0.22 \mathrm{eV}$ в диапазоне температур 0-36. $6^{\circ} \mathrm{C}$ и $0.135 \mathrm{eV}$ в диапазоне температур $\left.36.6-100^{\circ} \mathrm{C}\right)$ и почти на порядок превышает среднюю кинетическую энергию теплового движения молекул при нормальных условиях. Таким образом, предположение Рентгена [4] о двухкомпонентной структуре воды получает свое подтверждение. Явление сверхпластичности ряда металлов так же описывается именно с помощью гипотезы о существовании двухкомпонентной структуры [26].

Размеры кластеров в диапазоне температур 0-100 ${ }^{\circ} \mathrm{C}$ варьируют в 7.43 раза от 9.06 до $1.22 \mathrm{~nm}$. В связи с этим результаты малоуглового рассеяния излучения лазера оптического диапазона [11-13], где размеры неоднородностей структуры воды оцениваются от 0.3 до $6.0 \mu \mathrm{m}$, нужно интерпретировать как стабильные пузырьки растворенного газа (бабстоны).

Доля свободных молекул воды в диапазоне температур $0-100^{\circ} \mathrm{C}$ возрастает в 177 раз от значения 0.0104 до $1.84 \%$ соответственно. При температуре $\geq 36.6^{\circ} \mathrm{C}$ доля свободных молекул скачком возрастает в 24 раза от 0.027 до $0.64 \%$. Таким образом, в диапазоне температур, характерных для живых организмов, доля свободных молекул не превышает $1 \%$. Вместе с тем, согласно данным [6], в составе так называемых ассоциатов (кластеров) находится 70\% молекул воды и $30 \%$ в свободном состоянии. Аналогичные данные приведены в работе [8]. Полученные противоречия требуют дополнительных исследований.

На первый взгляд кажется, что количество свободных молекул воды слишком незначительно для ее течения. Вместе с тем процесс образования сверхпластичности титана при насыщении его водородом [27] также объясняется наличием „свободных нанокластеров“. Оказывается, что при объемной концентрации водорода 10\%, титан и его сплавы, имеющие поликристаллическую структуру, превращаются в квазижидкость, причем исследования с помощью электронного микроскопа показывают, что при сильном деформировании монокристаллические зерна титана распадаются на кластерные структуры размером порядка $100 \mathrm{~nm}$ с образованием гидридных частиц на границах этих структур [26]. Водород выступает в этом случае не в виде свободных молекул, а в виде физико-химических комплексных образований, т. е. относительная к общему числу атомов титана концентрация „частиц“ гидридов, обеспечивающих сверхпластическое скольжение, существенно ниже $12 \%$, что хорошо согласуется с нашими результатами для воды.

Время жизни молекулы воды в составе кластера убывает в 24.4 раза при повышении температуры и равно $3.9 \cdot 10^{-10} \mathrm{~s}$ в диапазоне температур $0-36.6^{\circ} \mathrm{C}$ и $1.6 \cdot 10^{-11} \mathrm{~s}$ в диапазоне температур $36.6-100^{\circ} \mathrm{C}$. Эти значения времени жизни существенно превышают величину $\sim 10^{-12} \mathrm{~s}[15]$, которая считается характеристикой среднего времени жизни водородных связей. В работе [15] это время считается временем жизни всего кластера воды, при этом не учитывается тот факт, что разрыв одной водородной связи не означает разрушения всего кластера. 
Полученное нами превышение времени жизни молекулы в кластере относительно среднего времени разрыва водородной связи [15] зависит от температуры. С одной стороны, наличие такой зависимости очевидно. С другой стороны, в диапазоне температур 0-36. $6^{\circ} \mathrm{C}$ это превышение составляет 390 раз, а в диапазоне $36.6-100^{\circ} \mathrm{C}-16$ раз. Столь существенное расхождение оценок времени жизни кластеров требует дальнейшего исследования.

Число молекул в составе кластеров воды в диапазоне температур $0-100^{\circ} \mathrm{C}$ убывает от 100000 до 250, соответственно. Эти результаты во много раз расходятся с результатами экспериментальных и теоретических исследований. Так, в работах $[10,14]$ устойчивыми считаются кластеры с числом молекул от 3 до 18. В работе [7] во всем диапазоне температур $\left(0-100^{\circ} \mathrm{C}\right)$ число молекул в кластере монотонно убывает от 30 до 9 штук. Вработе [8] стабильными считаются кластерные структуры с числом молекул 20, 57 и 912 во всем диапазоне температур от 0 до $100^{\circ} \mathrm{C}$. Обсуждается миграция протона $\left(\mathrm{H}^{+}\right)$в пределах кластера по эстафетному механизму. Этот процесс трудно объяснить с точки зрения соотношения энергий: теплового движения при нормальных условиях $\sim 0.03 \mathrm{eV}$, водородной связи в димере $\left(\mathrm{H}_{2} \mathrm{O}\right)_{2}-0.24 \mathrm{eV}[15]$ и диссоциации $\left(\mathrm{H}^{+}+\mathrm{OH}^{-}\right)-$ $4.8 \mathrm{eV}$. То есть процесс диссоциации молекулы воды (внутри кластера) маловероятен, что подтверждается известными данными о дистиллированной воде.

Причину расхождения данных, полученных в настоящей работе, с результатами работ, опубликованных в литературе, можно объяснить различиями методов изучения свойств воды. Единственные эмпирические параметры, использованные в настоящей работе - это температурные зависимости плотности и коэффициента вязкости воды. При измерениях коэффициента вязкости скорость направленного движения воды примерно в 1000 раз меньше скорости теплового движения самих молекул воды. Поэтому воздействием измерительной процедуры на кластеры воды можно пренебречь, чего нельзя сказать о других используемых для анализа кластерной структуры воды методах.

Оптические методы измерений - энергия кванта оптического диапазона имеет значение от 1.5 до $3.0 \mathrm{eV}$. Энергия квантов при рентгеновских способах исследования составляет $10 \mathrm{keV}$ и выше. Таким образом, при таких „энергичных“ способах измерений мы изучаем не сам кластер, а результат его взаимодействия с фотонами, электронами, нейтронами, энергия которых во много раз превышает не только энергию связи молекул воды с кластером $(0.22$ и $0.135 \mathrm{eV})$, но и энергию диссоциации молекулы $\mathrm{H}_{2} \mathrm{O}$. Образование ионов $\left(\mathrm{H}^{+}, \mathrm{OH}^{-}\right)$ приводит к огромным значениям электрических полей $\left(E \sim 1.5 \cdot 10^{11} \mathrm{~V} / \mathrm{m}\right)$, которые способны полностью разрушить исследуемые структуры воды.

Кроме того, пучки лазера, использованные при проведении исследований в работах [10-15], имеют мощность излучения на $1 \mathrm{~cm}^{2}$ поверхности, которая соответствует падению 1000 фотонов в секунду на каждую молекулу воды в ее поверхностном слое, что может приводить к развитию нелинейных многофотонных процессов.

Полученное в работе значение энергии связи молекулы воды с кластером $0.22 \mathrm{eV}$ очень близко к энергии водородной связи в димере $\left(\mathrm{H}_{2} \mathrm{O}\right)_{2}-0.24 \mathrm{eV}$ [15]. Приведем для сравнения значение теплоты плавления льда, приведенной к 1 молекуле: $E_{\text {melt }}(1)=0.0624 \mathrm{eV} / \mathrm{mol}$ [25]. Оказывается, что величина энергии связи молекулы воды с кластером в 3.5 раза превосходит значение теплоты плавления льда на одну молекулу. С учетом того что теплота плавления в некоторой степени определяет механическую прочность твердых тел [28], представление o „твердых“ кристаллитах обосновано. Этим фактом можно объяснить и слабую зависимость сжимаемости воды от давления [25].

Полученные результаты важны для некоторых технологических применений воды (электролиз, сверхъемкие аккумуляторы и конденсаторы $[29,30])$. Поперечный размер углеродных нанотрубок, используемых для существенного увеличения поверхности пластин электродов, составляет 5-8 nm при длине канала до десятков тысяч нанометров. Средний размер кластерных структур воды в диапазоне „рабочих“ температур от 0 до $40^{\circ} \mathrm{C}$ изменяется от 9.5 до $3.4 \mathrm{~nm}$. Он соизмерим с поперечным размером нанотрубок. При малой доле свободных молекул соотношение размеров кластера и диаметра нанотрубок не позволяет достаточно эффективно использовать всю их внутреннюю поверхность.

Важную роль полученные результаты о структуре воды играют в механизме обмена веществ в организме человека. Клетка покрыта мембраной. Вода является переносчиком питательных веществ внутрь клетки. Она же участвует в удалении „отработанных“ шлаков из клетки. Долгое время считалось, что обмен происходит за счет диффузии питательных веществ через мембрану внутрь клетки и шлаков - наружу. Порообразующий белок аквапорин [31] входит в состав мембраны. Сопоставим экспериментально измеренные параметры пор аквапорина со свойствами воды. Средний диаметр поры составляет $\sim 0.6 \mathrm{~nm}$, длина канала поры $\sim 8-10 \mathrm{~nm}$.

Размер кластера воды при температуре $36.6^{\circ} \mathrm{C}$, согласно нашим данным, превышает поперечный размер поры в $\sim 8$ раз [24,31]. Следовательно, обмен клетки с окружающей средой обусловлен только свободными молекулами воды.

Средняя длина свободного пробега молекулы $\mathrm{H}_{2} \mathrm{O}$ превышает поперечный размер поры клеточной мембраны в 6 раз. Следовательно, в канале поры - физический вакуум. При температуре $36.6^{\circ} \mathrm{C}$ скорость молекулы воды равна $655 \mathrm{~m} / \mathrm{s}$. При такой скорости время пробега молекулы воды вдоль канала поры в мембране составит порядка $1.5 \cdot 10^{-11} \mathrm{~s}$.

При значении потока молекул $\mathrm{H}_{2} \mathrm{O}$ через пору, равном $3 \cdot 10^{9} 1 / \mathrm{s}[31]$, средний временной интервал между молекулами составит $3.3 \cdot 10^{-10} \mathrm{~s}$. Следовательно, временной интервал между молекулами воды в 22 раза превышает 
время пролета молекулы сквозь канал поры в мембране. Таким образом, 95\% времени канал аквапорина пуст и возможен не только процесс поступления питательных веществ внутрь клетки, но и обратный процесс удаление отработанных шлаков из клетки через один и тот же канал.

Оценим, как механизм клеточного обмена на молекулярном уровне согласуется с макроскопическими параметрами водного обмена организма взрослого человека массой $75 \mathrm{~kg}$.

В состоянии покоя (без физической нагрузки) при значении пульса 60-80 ударов в минуту, сердце многократно перекачивает полный объем крови 5.51 (или $7.92 \mathrm{~m}^{3}$ в сутки). Секундный расход крови составит $92 \mathrm{~cm}^{3} / \mathrm{s}$, что в пересчете на воду, содержащуюся в крови, составит $48.1 \mathrm{~cm}^{3} / \mathrm{s}$. Число молекул воды, прокачиваемой за $1 \mathrm{~s}$, составит $1.6 \cdot 10^{24}$. Из всего количества молекул только малая доля $(0.63 \%)$ представлена в виде отдельных молекул. Таким образом, поток свободных молекул составит $1.23 \cdot 10^{22} 1 / \mathrm{s}$.

Поток молекул воды через пору аквапорина измерен экспериментально [31] и составляет $3 \cdot 10^{9} 1 / \mathrm{s}$. Следовательно, минимальное общее число пор аквапорина в организме человека должно составлять $4.1 \cdot 10^{12}$. Полное число клеток эритроцитов является стандартной величиной для здорового человека и составляет в среднем $4.75 \cdot 10^{12}$ на литр для мужчин и $4.25 \cdot 10^{12}$ на литр для женщин. Организм мужчины содержит в среднем 5.51 крови, женщины - 3.91 крови. Следовательно, организм мужчины содержит в среднем $26.1 \cdot 10^{12}$ эритроцитов, а организм женщины - $16.6 \cdot 10^{12}$ эритроцитов. За счет работы сердца полный объем крови прокачивается в организме за $60 \mathrm{~s}$, откуда получаем, что за одну секунду в процессе переноса питательных веществ участвует $0.435 \cdot 10^{12}$ эритроцитов в мужском и $0.28 \cdot 10^{12}$ эритроцитов в женском организме. Таким образом, суммарное число пор аквапорина, обеспечивающих нормальную жизнедеятельность организма, составляет $4.1 \cdot 10^{12} \mathrm{l} / \mathrm{s}$. Следовательно, каждый эритроцит должен содержать 9 пор для мужчин и 11 пор для женщин.

При физических нагрузках секундный расход крови возрастает примерно в 3 раза, во столько же раз возрастает и число клеток эритроцитов, участвующих в процессе обмена веществ за $1 \mathrm{~s}$. Вероятно, основной механизм заключается в увеличении интенсивности обмена через каждую пору за счет роста температуры крови.

Тренированный спортсмен во время соревнований за счет увеличения частоты пульса, объема и мощности сердца способен увеличить циркуляцию крови в 6.4 раза. Причем как минимум половину этого увеличения может обеспечить дополнительный объем крови, поступающий при физических нагрузках в кровеносную систему, а еще три раза набираются за счет интенсификации обмена в каждой поре.

Вероятнее всего, такая интенсификация имеет ограничения, которые могут стать предметом дальнейших исследований.

\section{Заключение}

Во всем диапазоне температур от 0 до $100^{\circ} \mathrm{C}$ вода имеет кластерную структуру. Оценки показывают, что основная доля молекул воды (до 99\%) находится внутри кластеров. Кластеры представляют собой твердые конструкции. Энергия связи молекулы воды с кластером в 23.5 раза превосходит теплоту плавления льда, отнесенную к одной молекуле. Время жизни молекулы в составе кластера существенно больше времени тепловых колебаний молекул в твердом теле. Таким образом, гипотеза Я.И. Френкеля [3] о характере теплового движения атомов (молекул) жидкости получает свое количественное обоснование. Подтверждена также идея Рентгена [4] о двухкомпонентной структуре воды.

Данные о повышенной прочности кластерных структур имеют большое значение для технологических приложений. Этот эффект нуждается в более подробном изучении.

Сопоставление размеров пор в клеточных мембранах с размером кластера воды показывает, что обмен веществ через поры носит молекулярный характер.

Полученные данные об относительно небольшом количестве свободных молекул хорошо согласуются с данными о механизмах сверхпластичности насыщенного водородом титана.

Исследование выполнено при поддержке программ Президиума РАН № 31.

\section{Список литературы}

[1] Данков П.Д., Кочетков А.А. // ДАН СССР. 1934. Т. 2. № 6. C. 359.

[2] Stewart G.W., Roger M. // Phys. Rev. 1927. Vol. 30. N 3. P. 232-244. DOI:10.1103/PhysRev.30.232

[3] Френкель Я.И. Кинетическая теория жидкостей. Изд-во АН СССР, М.,Л.: 1945.357 с.

[4] Röntgen W.C. // Annalen der Physik. 1892. Vol. 281. N 1. P. 91-97. DOI:10.1002/andp.18922810108

[5] Маленков Г.Г. // Журнал структурной химии. 2007. Т. 47. C. 5-35.

[6] Слесарев В.И. Химия. Основы химии живого. СПб. Химиздат, 2007. 784 с.

[7] Саргаева Н.П., Саргаев П.М. // Известия РГПУ им. А.И. Герцена, 2007. Т. 7. № 26. С. 112-126.

[8] Мосин О.В. // Электроника и связь. 2002. № 15. С. 24-29.

[9] Третьяков М.Ю. и др. // УФН. 2014. Т. 184. № 11. C. 1199-1215. DOI: 10.3367/UFNr.0184.201411c.1199 [Tretyakov M.Yu. et al. // Phys. Usp. 2014. Vol. 57. N 12. P. 1083-1098.]

[10] Moro R., Rabinovich R., Xia C., Kresin V.V. // Phys. Rev. Lett. 2006. Vol. 97. N 12. P. 123401-123404. DOI:10.1103/PhysRevLett.97.123401

[11] Коваленко В.Ф., Бордюк А.Ю., Шутов С.В. // Оптика атмосферы и океана. 2011. Т. 24. № 7. С. 601-605.

[12] Черникова Т.М., Иванов В.В., Михайлова Е.А. // Механика. 1997. Т. 38. № 1. С. 136-139. 
[13] Бункин Н.Ф., Суязов Н.В., Ципенюк Д.Ю. // Квант. электрон. 2005. Т. 35. № 2. С. 180-184. DOI: 10.1070/QE2005v035n02ABEH002898 [Bunkin N.F. et al. // Quant. Electron. 2005. Vol. 35. N 2. P. 180-184.]

[14] Саркисов Г.Н. // УФН. 2006. Т. 176. № 8. С. 833-845. DOI: 10.3367/UFNr.0176.200608b.0833 [Sarkisov G.N. // Phys. Usp. 2006. Vol. 49. P. 809-820.]

[15] Захаров С.Д., Мосягина И.В. Кластерная структура воды (обзор) // Препринт Физический ин-т им. П.Н. Лебедева PAH, 2011. 24 c.

[16] Barbour L.J., Orr G.W., Atwood J.L. // Nature. 1998. Vol. 393. N 6686. P. 671-673. DOI: 10.1038/31441

[17] Lang S.M. et al. // Phys. Chem. Chem. Phys. 2016. Vol. 18. N 23. P. $15727-15737$. DOI: $10.1039 / \mathrm{c} 6 \mathrm{cp} 00779 \mathrm{a}$

[18] Atrazhev V.V. et al. // J. The Electrochem. Society. 2017. Vol. 164. N 12. P. F1265-F1271. DOI: 10.1149/2.0041713jes

[19] Chaban V.V. // Phys. Chem. Chem. Phys. 2018. Vol. 20. N 3. P. 23754-23761. DOI: $10.1039 / \mathrm{c} 8 \mathrm{cp} 04012 \mathrm{e}$

[20] Verdaguer A. et al. // Chem. Rev. 2006. Vol. 106. N 4. P. 1478-1510. DOI: $10.1021 / \mathrm{cr} 0403761$

[21] Liu J. et al. // Chem. Sci. 2018. Vol. 9. P. 2065-2073. DOI:10.1039/C7SC04205A

[22] Хвольсон О.Д. Курс физики. Л.М.: Государственное технико-теоретическое издательство, 1933. 657 с.

[23] Хастед Джс. Физика атомных столкновений. М.: Мир, 1965. $465 \mathrm{c}$.

[24] Полянская А.В., Полянский А.М. // Тезисы докладов I Российской конф. „Физика - наукам о жизни“. СПб.: ФТИ им. А.Ф. Иоффе РАН, 2016. С. 91.

[25] Григорьев И.С., Мейлехов Е.3. Физические величины. Справочник. М.: Энергоатомиздат, 1991. 1232 с.

[26] Мурзинова А., Салищев Г.А., Афоничев Д.Д. // Физика металлов и металловедение. 2007. Т. 104. № 2. С. 204-211.

[27] Колачев Б.А. и др. // Технология легких сплавов. 2007. № 3. C. $10-26$.

[28] Polyanskiy A.M. et al. // Acta Mechanica. 2018. Vol. 229. N 12. P. 4863-4873. DOI:10.1007/s00707-018-2262-8

[29] Богданов А.А, Полянский А.М., Фих А.Я. // Сборник трудов IX Междунар. конф. „Современный физический практикум“. Волгоград. 2006. С. 134.

[30] Елецкий А.В., Зицерман В.Ю., Кобзев Г.А. // Технические науки - от теории к практикею Россия, Новосибирск, 2013. C. $105-112$.

[31] Murata K. et al. // Nature. 2000. Vol. 407. N 6804. P. 599-605. DOI:10.1038/35036519 